\title{
Differentiation of distal ureteral stones and pelvic phleboliths using a convolutional neural network
}

\author{
Johan Jendeberg $^{1}\left(\mathbb{D} \cdot\right.$ Per Thunberg $^{2}\left(\mathbb{D} \cdot\right.$ Mats Lidén $^{1} \mathbb{C}$
}

Received: 23 December 2019 / Accepted: 7 February 2020 / Published online: 27 February 2020

(c) The Author(s) 2020

\begin{abstract}
The objectives were to develop and validate a Convolutional Neural Network (CNN) using local features for differentiating distal ureteral stones from pelvic phleboliths, compare the CNN method with a semi-quantitative method and with radiologists' assessments and to evaluate whether the assessment of a calcification and its local surroundings is sufficient for discriminating ureteral stones from pelvic phleboliths in non-contrast-enhanced CT (NECT). We retrospectively included 341 consecutive patients with acute renal colic and a ureteral stone on NECT showing either a distal ureteral stone, a phlebolith or both. A 2.5 -dimensional CNN (2.5D-CNN) model was used, where perpendicular axial, coronal and sagittal images through each calcification were used as input data for the CNN. The CNN was trained on 384 calcifications, and evaluated on an unseen dataset of 50 stones and 50 phleboliths. The CNN was compared to the assessment by seven radiologists who reviewed a local $5 \times 5 \times 5 \mathrm{~cm}$ image stack surrounding each calcification, and to a semi-quantitative method using cut-off values based on the attenuation and volume of the calcifications. The CNN differentiated stones and phleboliths with a sensitivity, specificity and accuracy of $94 \%, 90 \%$ and $92 \%$ and an AUC of 0.95 . This was similar to a majority vote accuracy of 93\% and significantly higher $(p=0.03)$ than the mean radiologist accuracy of $86 \%$. The semi-quantitative method accuracy was $49 \%$. In conclusion, the CNN differentiated ureteral stones from phleboliths with higher accuracy than the mean of seven radiologists' assessments using local features. However, more than local features are needed to reach optimal discrimination.
\end{abstract}

Keywords Computed tomography $\cdot$ Ureteral calculi $\cdot$ Pelvic phlebolith $\cdot$ Deep learning $\cdot$ Convolutional neural networks

$\begin{array}{ll}\text { Abbreviations } \\ \text { NECT } & \begin{array}{l}\text { Non-contrast-enhanced computed } \\ \text { tomography }\end{array} \\ \text { CTU } & \text { CT urography } \\ \text { CNN } & \begin{array}{l}\text { Convolutional neural network } \\ \text { 2.5D-CNN }\end{array} \\ & \begin{array}{l}2.5 \text {-Dimensional CNN including axial, coro- } \\ \text { nal and sagittal images }\end{array} \\ \text { CAD } & \text { Computer-aided diagnosis } \\ \text { ANN } & \text { Artificial neural network } \\ \text { AI } & \text { Artificial intelligence } \\ \text { FC layer } & \text { Fully connected layer } \\ \text { PACS } & \text { Picture archiving and communication system } \\ \text { MPR } & \text { Multiplanar reconstructions } \\ \text { CI } & \text { Confidence interval }\end{array}$

Johan Jendeberg

johan.jendeberg@gmail.com

1 Department of Radiology, Faculty of Medicine and Health, Örebro University Hospital, 70185 Örebro, Sweden

2 Department of Medical Physics, Faculty of Medicine and Health, Örebro University, Örebro, Sweden
ROC Receiver operating characteristic

AUC Area under the curve

ROI Region of interest

\section{Key points}

A Convolutional Neural Network for classifying pelvic calcifications was developed and validated.

The Convolutional Neural Network differentiated ureteral stones from phleboliths with higher accuracy than the mean assessment by seven radiologists.

More than local image features are needed to correctly classify pelvic calcifications 


\section{Introduction}

For more than 20 years, non-contrast enhanced computed tomography (NECT) has been the examination of choice for diagnosing ureteral stones. At detection, about two thirds $(62-68 \%)$ of all ureteral stones are positioned in the lower part of the ureter (defined as overlying or below the sacroiliac joint) [1-3]. For a number of reasons, the assessment of this part of the ureter is a challenge even for an experienced radiologist. The lower ureters are located close to blood vessels, bowels and adnexa and, in lean patients in particular with a small amount of intra-abdominal fat, it may be impossible to separate those structures and identify a non-dilated distal ureter.

A frequent finding in the pelvis is phleboliths or wall calcifications of small veins. They have a prevalence of about $40 \%$ in the adult population [4], can be located close to the ureters and can be hard to distinguish from a distal stone. CT urography (CTU) to delineate the ureters is one method used to determine whether the calcification is a stone or a phlebolith. However, CTU has the disadvantage of increased radiation exposure and exposure to potentially nephrotoxic iodinized contrast media. Different approaches based on local features have been tried to distinguish urinary stones from phleboliths, such as the "soft tissue rim sign" [5-8] used to indicate a stone in the ureter and "the comet sign", a central lucency or low attenuation $[6,9]$, used to indicate a phlebolith. In everyday practice, these signs are often insufficient for differential diagnosis, Fig. 1.

Another approach for differentiating stones from phleboliths is Computer-Aided Diagnosis (CAD). Differentiation using these methods is based on automatically or semi-automatically derived local image features of the calcifications.
Recently, a semi-quantitative method was applied using cutoff values for the volume and attenuation of the calcification to discriminate stones from phleboliths [10]; while another method used image features that were fed into an artificial network [11]. Irrespective of the CAD method used, a key question for their development is whether the images of the calcification and its local surroundings can provide sufficient information for differentiation, or whether distant information, such as from visible upper ureters, is also needed.

Recent years have seen an enormous interest in artificial neural network (ANN)-based artificial intelligence (AI) in medical imaging $[12,13]$. Briefly, an ANN is built from a simple mathematical nerve cell model, a neuron, which computes one output value from multiple input values. When a large number of neurons are arranged in inter-connected layers, the ANN can be optimized-trained-to predict outcomes based on the input of the first layer. A convolutional neural network (CNN) is an ANN with a specific arrangement in one or more layers called convolutional layers, which are especially suited for image analysis. A CNN can be fed with annotated images and learn to classify them through automatic iterative adjustments of the weighted neuron functions [14].

To the best of our knowledge, there are no previous studies that evaluate a $\mathrm{CNN}$ method for differentiating stones from phleboliths.

Therefore, with the overall aim of evaluating whether the assessment of a calcification and its surroundings is sufficient for discriminating distal ureteral stones from pelvic phleboliths in NECT, the objectives of this study were to: (1) Develop and validate a CNN method using local features for differentiating distal ureteral stones from pelvic phleboliths. (2) Compare the CNN method with a semi-quantitative

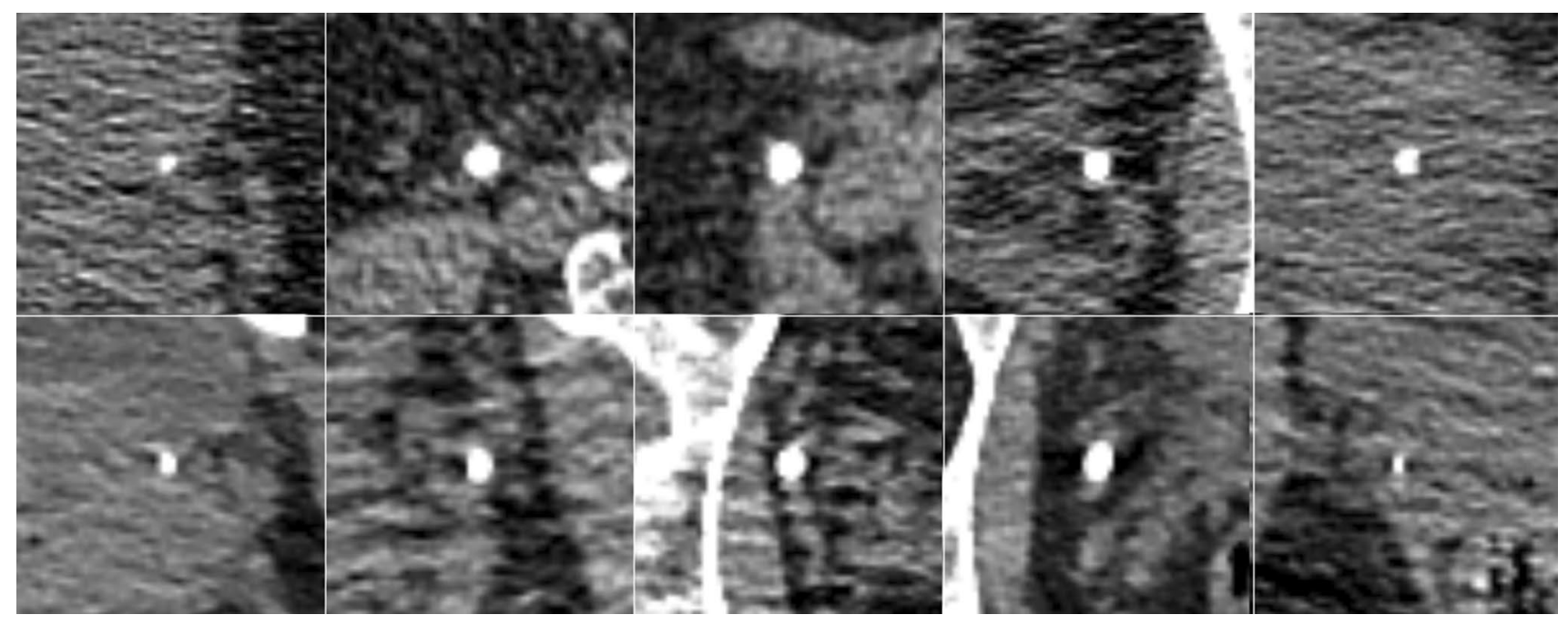

Fig. 1 Examples of pelvic calcifications. Upper row Distal ureteral stones, Lower row Phleboliths 
method, and with radiologists' assessments of the calcification and its local surroundings.

\section{Methods}

The Regional Research Ethics Board approved this retrospective study and waived informed consent. We reviewed 1824 consecutive, acute NECT in patients referred in the period April 2012-September 2014 from the local Emergency Department with symptoms of suspected acute ureteral stone.

Inclusion and exclusion numbers and criteria are shown in Fig. 2. Sample size was defined according to a previous study using the same material [3] and therefore, no specific power analysis for the present study was performed.
From the retrospectively created data bank, 341 patients with a stone in the lower ureter $(n=267)$ and/or a pelvic phlebolith $(n=217)$ were included. We created a pseudonymized image stack of $1-\mathrm{mm}$ slices around each calcification as a cube measuring $5 \times 5 \times 5 \mathrm{~cm}$ (fifty 1 -mm images with a limited field of view of $5 \times 5 \mathrm{~cm}$ ) for use in the study.

The stone group consisted of 65 women and 202 men, with a mean age of 49 years (range 18-100). The phlebolith group included 49 women and 168 men; the mean age was 53 years (range 22-86). Average stone size (largest diameter) was $4.5 \mathrm{~mm}$ (range $2.0-11.8 \mathrm{~mm}$ ) and average phlebolith size $4.5 \mathrm{~mm}$ (range $2.8-9.6 \mathrm{~mm}$ ). 110 stones and 91 phleboliths were examined using a 40-detector row CT scanner (Brilliance, Philips Medical Systems Best, The Netherlands) with a low-dose NECT protocol for the urinary tract $(120 \mathrm{kV}, 70 \mathrm{mAs} / \mathrm{slice}, \mathrm{CTDI} 4.9 \mathrm{mGy}, 40 \times 0.625 \mathrm{~mm}$,

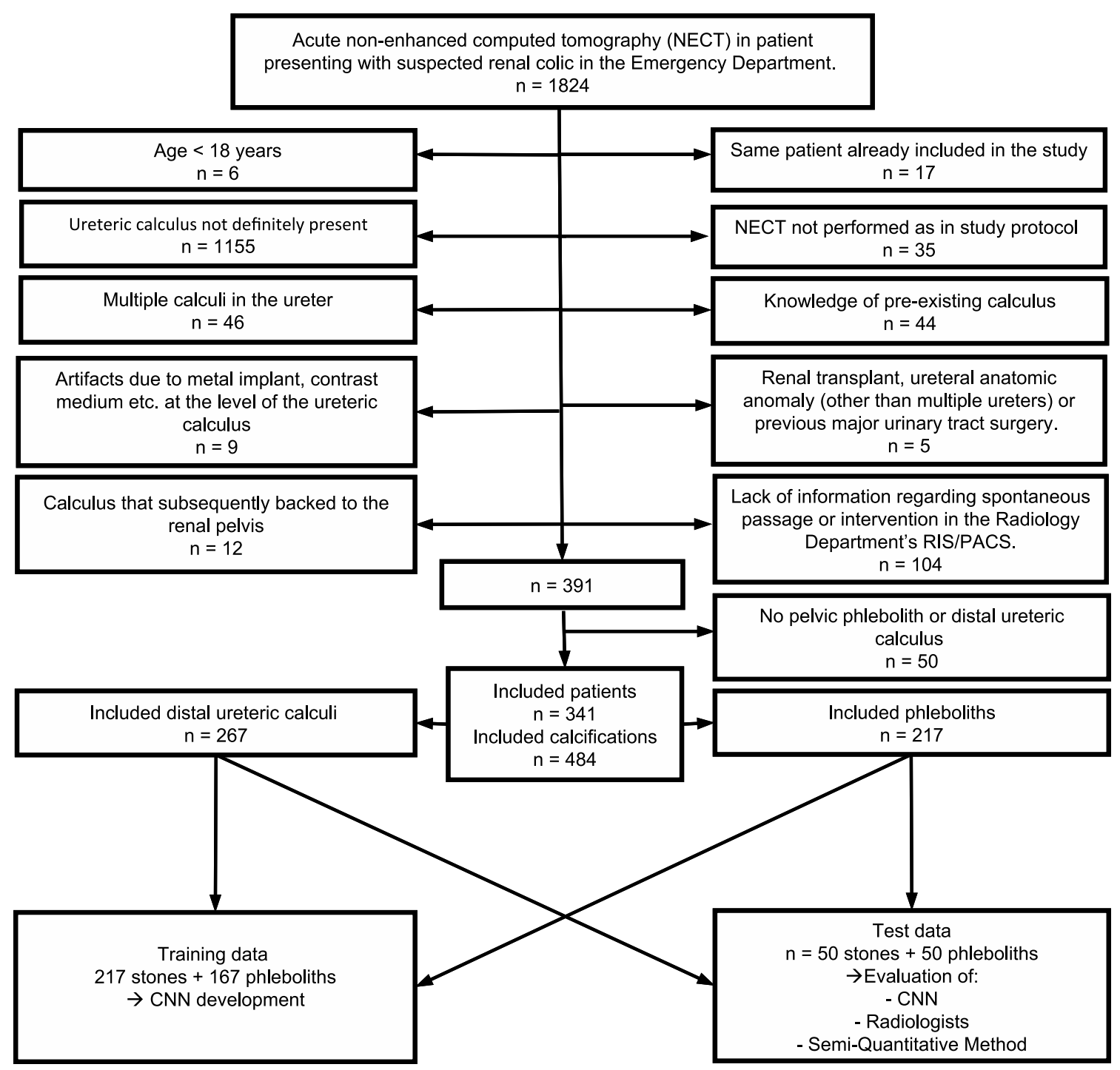

Fig. 2 Flowchart of inclusion and exclusion criteria. NECT non-contrast enhanced computed tomography, $C N N$ convolutional neural network 
standard filter [B], supine position). 157 stones and 126 phleboliths were examined using a $2 \times 128$-channel scanner (Somatom Definition Flash, Siemens, Erlangen, Germany) $(120 \mathrm{kVp}, 70 \mathrm{mAs} / \mathrm{slice}$ CTDI $4.7 \mathrm{mGy} 128 \times 0.6 \mathrm{~mm}$, filter B20f, B25f or I30f, supine position).

\section{Ground truth}

At inclusion, one radiologist (with 12 years' experience of reading abdominal $\mathrm{CT}$ ) used the complete diagnostic NECT examination, as well as knowledge from all follow-up examinations until stone passage, potential previous examinations and the clinical information in the referral, to diagnose the ureteral stones. Ipsilateral hydronephrosis, hydroureter, perinephric and periureteral stranding and the clinical information, as site of pain, were used for guiding to a distal ureteral stone, which had to be clearly visible in the distal ureter to be included.

When a pelvic phlebolith was present, the one most likely to be mistaken for a distal ureteral stone was included in the study. This assessment was subjective, but based on the size and nearness to the distal part of one the ureters. When necessary, several prior and subsequent examinations were used to define a phlebolith as such.

If there was doubt of the classification into a distal ureteral stone or a pelvic phlebolith, the calcification was not included.

\section{Test dataset}

As illustrated in the flowchart (Fig. 2), the stacks with calcifications were randomly split into two separate datasets. We created one smaller dataset containing 50 stones and 50 phleboliths from the included calcifications. This dataset was not used for training the neural network, but served as a validation dataset when evaluating the three methods for their ability to differentiate phleboliths from stones.

\section{Convolutional neural network (2.5D-CNN)}

A convolutional neural network was developed and trained using the training dataset comprised of the remaining 217 ureteral stones and 167 phleboliths. A 2.5-dimensional CNN (2.5D-CNN) model was used, where three perpendicular 2D images (axial, coronal and sagittal) were created having each calcification voxel as intercept. The image triplets for all voxels were used as input data for the CNN [15]. Each calcification was segmented using a threshold of $250 \mathrm{HU}$, and any hole in the segmented volume was filled using a morphologic operation.

The training data were augmented by mirroring in the left-right plane, where the anatomic differences are small.
Using each voxel location as a separate training example, each calcification contributed from a few to more than one thousand training examples, depending on its size. In total, the 384 stones and phleboliths generated 38068 training examples, i.e., image triplets.

Several different 2.5D-CNN architectures were tested on the training data and three different network candidates were selected, see Fig. 3. After selection, the three candidate networks were trained on the full training cohort.

For classification on the unseen test set, each calcification was segmented and 2.5D images generated similarly as in the training set, but without mirroring. The classification was performed on each voxel location in the calcification, and the output for each calcification was computed as the average probability output of the softmax classification layer. A cut-off of 0.5 on the final score was used for classification as stone or phlebolith.

\section{Radiologist assessments}

Seven radiologists, with 10-28 years of experience reading abdominal CTs independently reviewed the one hundred $5 \times 5 \times 5 \mathrm{~cm} 1-\mathrm{mm}$ stacks in the test data, using standard PACS (IDS7 Sectra AB, Linköping, Sweden). The readers were allowed to use all available features in the system, such as multiplanar reformats (MPR), zoom, attenuation and size measurements. The readers were blinded to the result of the reference standard, clinical information and distant image information (such as hydronephrosis, perirenal fat stranding etc.).

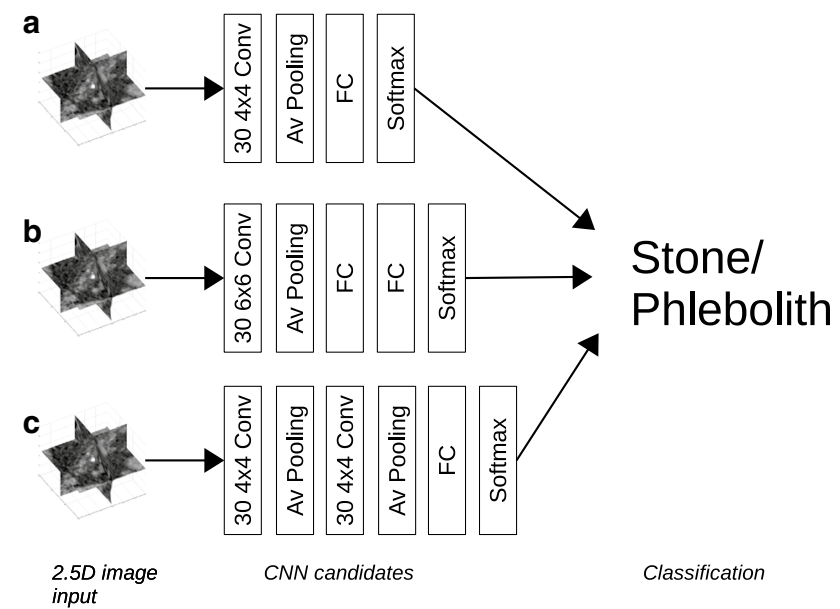

Fig. 3 Schematic architecture of the three 2.5D convolutional network (2.5D-CNN) candidates validated on the test dataset. Conv convolutional layer, $A v$ average pooling layer, $F C$ fully connected layer 


\section{Semi-quantitative method using attenuation and volume}

Following the protocol for a recently published study [10], classification of distal ureteral stones and phleboliths was performed based on the cut-off values below:

Attenuation $>643 \mathrm{HU} \rightarrow$ Ureteral stone.

Attenuation $<643 \mathrm{HU} \rightarrow$ Phlebolith.

Volume $>171 \mathrm{~mm}^{3} \rightarrow$ Ureteral stone.

Volume $<171 \mathrm{~mm}^{3} \rightarrow$ Phlebolith.

The same window settings (W300/L40) and slice thickness of $5 \mathrm{~mm}$ as in the original study were used for the measurements for the semi-quantitative method. The 5-mm slices were created as MPR based on 1-mm images. The volume of the calcification was calculated with the ellipsoid formula used in the original article, $\mathrm{W} \times \mathrm{L} \times \mathrm{H} \times \mathrm{Pi} / 6$, where $\mathrm{W}, \mathrm{L}$ and $\mathrm{H}$ are the lengths of the three principal axes of the ellipsoid. The mean attenuation was measured in the center of the calcification using the in PACS integrated tool for attenuation measurement, the region of interest (ROI) circle.

The size measurements of the calcifications were performed manually by a radiologist with 14 years' experience reviewing abdominal CTs. He was blinded to the results of the reference test, distant image information and clinical information and to the results of the assessments by the seven radiologists.

\section{Statistics}

Statistical analysis was performed using IBM SPSS for Mac OS v24.0.0.0 (SPSS Inc. Chicago, Il. USA) and Matlab R2018b (The Mathworks Inc, Natick, Mass, USA). The neural networks were developed using Matlab Deep Learning Toolbox.

Sensitivity, specificity and accuracy with $95 \%$ confidence intervals (CI) using binomial distribution were calculated for the neural network and for the radiologists' classifications compared to the reference standard. The average accuracy for the seven readers was calculated as well as the "vote majority", defined as the assessment of each calcification chosen by the majority of the readers $(\geq 4)$.

The statistical significance of the difference between the $\mathrm{CNN}$ and the mean accuracy of the readers was tested using the one sample $t$ test.

In the semi-quantitative method, the differences in volume and attenuation between stones and phleboliths were not normally distributed and were, therefore, analyzed using the Mann-Whitney $U$-test. The scatter plot was visually analyzed for alternative cut-off values for the semiquantitative method. Where applicable, the area under the Receiver Operating Characteristic (ROC) curve (AUC) was computed.

\section{Results}

\section{Validation on test dataset}

Neither the radiologists, nor the 2.5D-CNN, nor the semiquantitative method could fully differentiate between the pelvic calcifications in the test dataset. Cross tabulations for the three methods against the reference standard are shown in Table 1.

Table 1 Cross tabulations and accuracy with 95\% CI for three methods for differentiation of 50 distal ureteral stones and 50 pelvic phleboliths using only local features

\begin{tabular}{|c|c|c|c|c|}
\hline & \multicolumn{4}{|c|}{ Reference standard } \\
\hline & & Phlebolith & Stone & Total \\
\hline \multicolumn{5}{|c|}{ (a) 2.5D-CNN Prediction Stone vs phlebolith } \\
\hline \multirow[t]{3}{*}{$2.5 \mathrm{D}-\mathrm{CNN}$} & Phlebolith & 45 & 3 & 48 \\
\hline & Stone & 5 & 47 & 52 \\
\hline & Total & 50 & 50 & 100 \\
\hline \multicolumn{5}{|c|}{ Accuracy $92 \%$ (95\% CI 85-97\%) } \\
\hline \multicolumn{5}{|c|}{ (b) Cooperation all readers Prediction Stone vs phlebolith } \\
\hline \multirow{3}{*}{$\begin{array}{l}\text { Majority of the } \\
\text { readers assess- } \\
\text { ments }\end{array}$} & Phlebolith & 49 & 6 & 55 \\
\hline & Stone & 1 & 44 & 45 \\
\hline & Total & 50 & 50 & 100 \\
\hline \multicolumn{5}{|c|}{ Accuracy 93\% (95\% CI 86-97\%) } \\
\hline \multicolumn{5}{|c|}{ (c) Median reader assessment } \\
\hline \multirow[t]{3}{*}{ Median reader } & Phlebolith & 44 & 5 & 49 \\
\hline & Stone & 6 & 45 & 51 \\
\hline & Total & 50 & 50 & 100 \\
\hline \multicolumn{5}{|c|}{ Accuracy $89 \%$ (95\% CI 81-94\%) } \\
\hline \multicolumn{5}{|c|}{ (d) Semi-quantitative method: $\leq 643 \mathrm{HU}$ and $\leq 173 \mathrm{~mm}^{3} \rightarrow$ Phlebolith } \\
\hline \multirow{3}{*}{$\begin{array}{l}\text { Quantitative } \\
\text { prediction } \\
\text { volume + HU }\end{array}$} & Phlebolith & 38 & 39 & 77 \\
\hline & Stone & 12 & 11 & 23 \\
\hline & Total & 50 & 50 & 100 \\
\hline \multicolumn{5}{|c|}{ Accuracy $49 \%$ (95\% CI 39-59\%) } \\
\hline
\end{tabular}

Reference standard $=$ Calcification assessment by a radiologist using the complete NECT examination as well as knowledge from followup examinations until stone passage and clinical information

(a) Convolutional neural network (2.5D-CNN) using the information from perpendicular axial, coronal and sagittal images intersecting each voxel of the calcification

(b) Majority vote of seven radiologists using only a $5 \times 5 \times 5 \mathrm{~cm}$ large 1-mm slice stack surrounding the calcification

(c) Median radiologist assessment using only a $5 \times 5 \times 5 \mathrm{~cm}$ large 1-mm slice stack surrounding the calcification

(d) Semi-quantitative method using only the volume $\left(\mathrm{mm}^{3}\right)$ and attenuation (HU) of each calcification in a 5-mm slice stack 


\subsection{D-CNN}

There were only small differences in classification accuracy for the pelvic calcifications between the three candidate networks.

The accuracy on the unseen test set was $93 \%, 90 \%$ and 92\% and the AUC was 0.93, 0.93 and 0.95 for the three 2.5D-CNN candidates, respectively. For the further analysis, the third network candidate (Fig. 3c), with two convolutional layers, was selected. The sensitivity, specificity and accuracy for the third 2.5D-CNN candidate were 94\% (95\% CI 87-98\%), 90\% (95\% CI 82-95\%) and 92\% (95\% CI $85-97 \%)$, respectively.

\section{Radiologist assessment}

The average accuracy for the seven radiologists' classifications was $86 \%$ (range 76-91\%), which was significantly lower $(p=0.03)$ than the accuracy of the third 2.5D-CNN. Using a majority vote among the readers (classification of each calcification chosen by $\geq 4$ readers) for the assessments of the calcifications, the accuracy rose to $93 \%$ (95\% CI 86-97\%), sensitivity $88 \%$ (95\% CI 80-94\%) and specificity 98\% (95\% CI 93-100\%). The accuracy of the reader with the median result was $89 \%$ (95\% CI 81-94\%). The ROC curve for the third 2.5D-CNN compared to the radiologists' results is shown in Fig. 4.

\section{Semi-quantitative method}

There was no significant difference in the median volume $(p=0.70)$ or the median attenuation $(p=0.29)$ between the lower ureteral stones and pelvic phleboliths.

The AUC for classifying a pelvic calcification as a stone or phlebolith using the semi-quantitative method was 0.56 (95\% CI $0.45-0.68)$ and 0.52 (95\% CI 0.41-0.64) for the attenuation and volume measured on 5-mm images, respectively. Use of the proposed cut-off values of $643 \mathrm{HU}$ and $171 \mathrm{~mm}^{3}$ did not enable differentiation between a stone and a phlebolith, see Fig. 5 and Table $1 \mathrm{~d}$.

The scatter plot did not reveal an alternative cut-off suitable for differentiation between a ureteral stone and phlebolith.

\section{Discussion}

In this study, we showed that a convolutional neural network (2.5D-CNN) that had been trained on a dataset of 384 pelvic calcifications, using only local image features, could classify calcifications in an unseen test set into lower ureteral stones and phleboliths with an accuracy of more than $90 \%$. This result was similar to the majority vote among seven

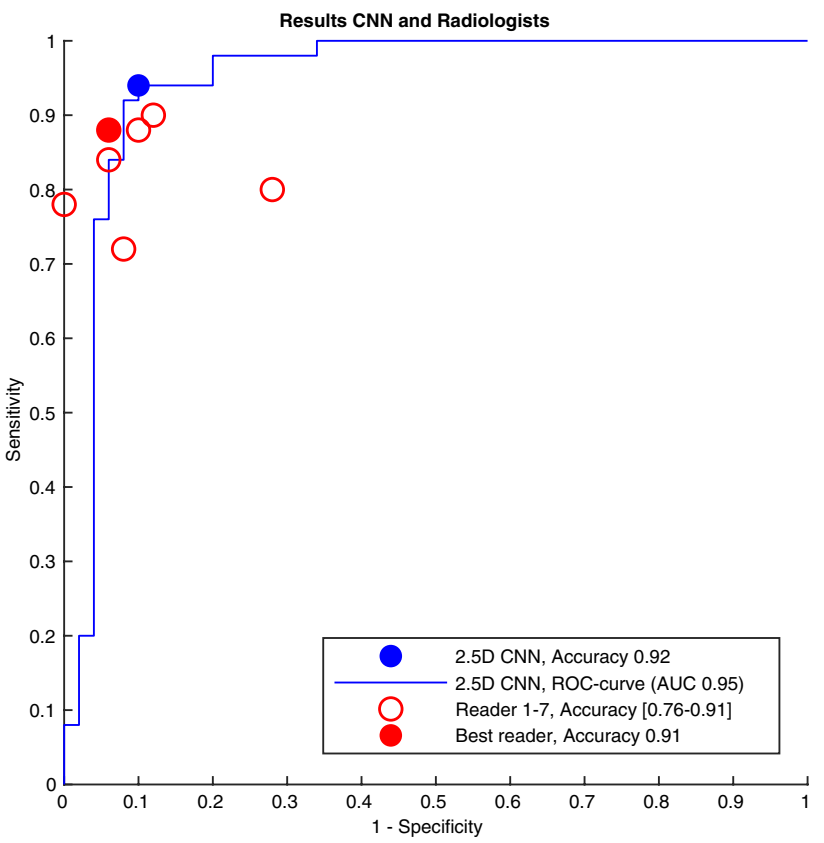

Fig. 4 Reciever operator characteristics (ROC) curve for a convolutional neural network candidate compared to the accuracy of the assessements by seven radiologists

radiologists, also using only local image features, having a pooled accuracy of $93 \%$, and significantly better than the mean of the same radiologists' results.

In recent years, a large number of studies have been performed using machine learning in radiology in many different applications including neuroimaging and imaging of the chest and abdomen, where the main interest has been towards oncology imaging and anatomy [12]. In two previous studies, CNNs have been used for the detection of ureteral stones $[16,17]$. We are not aware of any study previously published on the differentiation between lower ureteral stones and pelvic phleboliths on NECT using CNN.

In 2010, Lee et al. [11] created an artificial neural network (ANN) that used combinations of various shape and internal texture parameters of 112 calcifications to classify them into ureteral stones or vascular calcifications. They reached an AUC of 0.85 for the shape parameters and 0.88 for texture parameters. However, the same study group was used for the ANN development and for validation, and their true AUC on a separate test set must be presumed to be lower.

To the best of our knowledge, the accuracy of $92 \%$ and an AUC of 0.95 on the unseen test set for the third candidate $2.5 \mathrm{D}-\mathrm{CNN}$ in our study is the best published result for this computer-aided diagnosis (CAD) application. These results are promising for the development of CAD for pelvic calcifications but, to be clinically useful, greater accuracy is necessary than that expected from an experienced radiologist 
Fig. 5 Scatter plot with manual measurements of volume and attenuation. $X$-axis: Mean attenuation (mean $\mathrm{HU}), Y$-axis: Volume $\left(\mathrm{mm}^{3}\right)$, Red circles Distal ureteral stones. Black stars Phleboliths

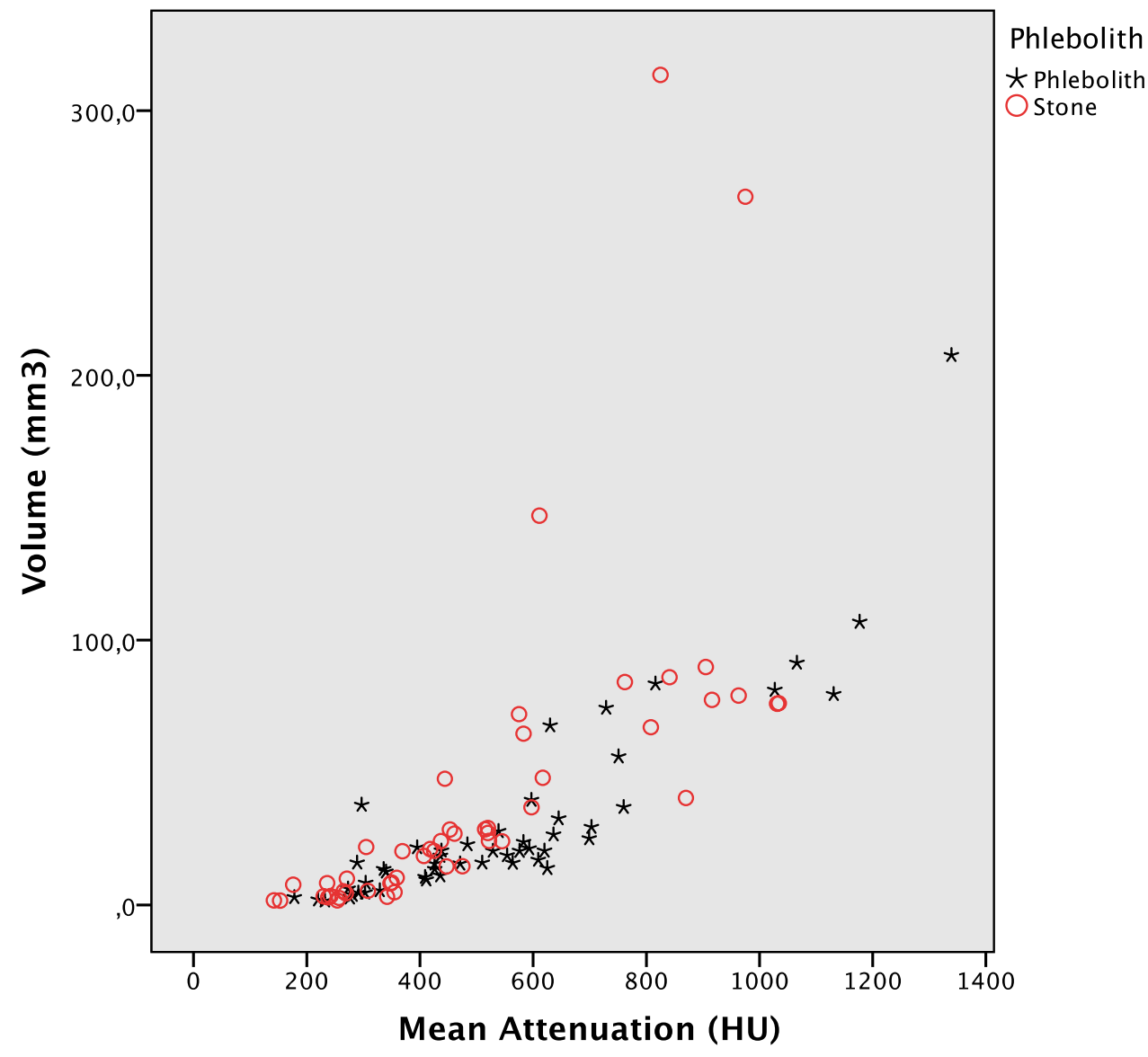

using the information contained in the complete NECT. It is also of major importance that ureteral stones are not falsely classified as phleboliths, which was the case in three out of 50 stones $(6 \%)$ in our test data set.

Neither the individual radiologists, nor the majority vote among the radiologists, nor the 2.5D-CNN could completely differentiate between stones and phleboliths using only local features in a $5 \times 5 \times 5 \mathrm{~cm}$ large cube surrounding the calcifications. This finding strongly suggests that local features are not sufficient for this discrimination and that future CNN models should contain distant information, such as all parts of the urinary tract and its closest surroundings for improved performance [17]. In daily practice, the radiologists assess various information such as hydronephrosis, hydroureter, perirenal or periureteral fat stranding, and information in the referral about ipsi- or contralateral symptoms together with the regional image information close to the calcification. Analogous to the performance of human readers, it is reasonable to believe that inclusion of more information sources in the ANN could improve the machine learning performance.

Earlier studies on visual local features and signs have shown divergent results, with mostly high specificity as, for example, $92-100 \%[6,9]$ for the rim sign indicating a ureteral stone and up to $100 \%$ for the comet sign [5, 7-9] indicating a phlebolith. The sensitivity has been lower, $50-77 \%$ and $21-65 \%$, respectively, and the authors have recommended the use of distant and clinical information along with the local features for the assessment of pelvic calcifications. In a study of Rochester Guest et al. the rim sign was accompanied by distant features of obstruction in all cases but one and the same study also found a pseudocomet sign in some of the ureteral stones [18]. The central lucency that was used to differentiate phleboliths from stones on plain radiographs has shown to be present only occasionally (1-9\%) on NECT [9, 19].

The ability of a recently published semi-quantitative method that used the attenuation and volume of the calcifications to differentiate between stone and phlebolith could not be confirmed in our study. The volume criterion in the semi-quantitative method is highly sensitive for inclusion criteria [20] and, similar to us, Bell et al. [9] did not find a significant difference in size between distal ureteral stones and phleboliths. In our study, we included the phlebolith most likely to be mistaken for a ureteral stone, as we considered that the radiologist or urologist would need automated assistance with this calcification. This might have contributed to the lack of difference in 
volume and attenuation between ureteral stones and phleboliths in our material.

Our study has limitations. Even though we used the largest dataset with labeled pelvic calcifications that has been used for machine learning so far, more training examples might have increased the accuracy of the CNN. A generalization of the $\mathrm{CNN}$ method would also require training examples from a wide range of scanners with different protocol parameters.

In regard to the inclusion of phleboliths, only proven phleboliths (proven with the help of consecutive examinations, distance NECT information, etc.) were included. This might have resulted in a bias towards a better accuracy than achievable in daily practice. We endeavored to minimize this bias by selecting the phlebolith most likely to be mistaken for a stone. Inclusion was performed retrospectively and only a very small number of the patients underwent ureteroscopy, which could, therefore, not be used for defining ground truth. However, we do not regard the lack of ureteroscopy as reference standard to be a major limitation. Ureteroscopy has two disadvantages compared to multiple radiologic followup examinations. Firstly, it cannot confirm the presence of a phlebolith, merely the presence of a ureteral stone. Secondly, spontaneous stone passage in the interval between the CT examination and the ureteroscopy could lead to false-negative results of the ureteroscopy.

In conclusion, we demonstrated that a $2.5 \mathrm{D}-\mathrm{CNN}$ could differentiate ureteral stones from phleboliths with a $92 \%$ accuracy, which is higher than the mean accuracy of assessment by seven radiologists. This finding suggests that AI can become a valuable tool for ureteral stone imaging. In contrast, the semi-quantitative method had a significantly lower accuracy. Importantly, neither the $\mathrm{CNN}$, nor the majority vote by seven trained readers was entirely accurate, which suggests that more than local image features, such as information from the complete CT examination and clinical information, is needed for the discrimination between distal ureteral stones and pelvic phleboliths.

Acknowledgments The authors thank the participating readers from the Department of Radiology, Örebro University Hospital. Open access funding provided by Örebro University.

Funding Johan Jendeberg received funding from Region Örebro Län, No: OLL-811941. Mats Lidén received funding from Region Örebro Län, No: OLL-684531. Per Thunberg recieved funding from Nyckelfonden, No: OLL-787911.

\section{Compliance with ethical standards}

Conflict of interest The authors of this manuscript declare no relationship with any companies, whose products or services may be related to the subject matter of the article.
Ethical approval This retrospective study was performed in accordance with the ethical standards of the Regional Research Committee and with the 1964 Helsinki declaration and its later amendments. The Regional Research Ethics Board approved the study protocol and waived the informed consent requirement.

Open Access This article is licensed under a Creative Commons Attribution 4.0 International License, which permits use, sharing, adaptation, distribution and reproduction in any medium or format, as long as you give appropriate credit to the original author(s) and the source, provide a link to the Creative Commons licence, and indicate if changes were made. The images or other third party material in this article are included in the article's Creative Commons licence, unless indicated otherwise in a credit line to the material. If material is not included in the article's Creative Commons licence and your intended use is not permitted by statutory regulation or exceeds the permitted use, you will need to obtain permission directly from the copyright holder. To view a copy of this licence, visit http://creativecommons.org/licenses/by/4.0/.

\section{References}

1. Coll DM, Varanelli MJ, Smith RC (2002) Relationship of spontaneous passage of ureteral calculi to stone size and location as revealed by unenhanced helical CT. AJR Am J Roentgenol 178:101-103. https://doi.org/10.2214/ajr.178.1.1780101

2. Song H-J, Cho S-T, Kim K-K (2010) Investigation of the location of the ureteral stone and diameter of the ureter in patients with renal colic. Korean Urol Assoc 51:198-201. https://doi. org/10.4111/kju.2010.51.3.198

3. Jendeberg J, Geijer H, Alshamari M et al (2017) Size matters: The width and location of a ureteral stone accurately predict the chance of spontaneous passage. Eur Radiol. https://doi. org/10.1007/s00330-017-4852-6

4. Luk ACO, Cleaveland P, Olson L et al (2017) Pelvic phlebolith: a trivial pursuit for the urologist? J Endourol 31:342-347. https ://doi.org/10.1089/end.2016.0861

5. Smith RC, Verga M, Dalrymple N et al (1996) Acute ureteral obstruction: value of secondary signs on helical unenhanced CT. Am J Roentgenol 167:1109-1113. https://doi.org/10.2214/ ajr.167.5.8911160

6. Boridy IC, Nikolaidis P, Kawashima A et al (1999) Ureterolithiasis: value of the tail sign in differentiating phleboliths from ureteral Calculi at nonenhanced helical CT. Radiology 211:619-621. https://doi.org/10.1148/radiology.211.3.r99ma 44619

7. Kawashima A, Sandler CM, Boridy IC et al (1997) Unenhanced helical CT of ureterolithiasis: value of the tissue rim sign. AJR Am J Roentgenol 168:997-1000. https://doi.org/10.2214/ ajr.168.4.9124157

8. Heneghan JP, Dalrymple NC, Verga M et al (2014) Soft-tissue "rim" sign in the diagnosis of ureteral calculi with use of unenhanced helical CT. Radiology 202:709-711. https://doi. org/10.1148/radiology.202.3.9051021

9. Bell TV, Fenlon HM, Davison BD et al (1998) Unenhanced helical CT criteria to differentiate distal ureteral calculi from pelvic phleboliths. Radiology 207:363-367. https://doi.org/10.1148/ radiology.207.2.9577482

10. Tanidir Y, Sahan A, Asutay MK et al (2017) Differentiation of ureteral stones and phleboliths using Hounsfield units on computerized tomography: a new method without observer bias. Urolithiasis 45:323-328. https://doi.org/10.1007/s00240-016-0918-1 
11. Lee HJ, Kim KG, Il HS et al (2010) Differentiation of urinary stone and vascular calcifications on non-contrast CT images: an initial experience using computer aided diagnosis. J Digit Imaging 23:268-276. https://doi.org/10.1007/s10278-009-9181-0

12. Soffer S, Ben-Cohen A, Shimon O et al (2019) Convolutional neural networks for radiologic images: a radiologist's guide. Radiology. https://doi.org/10.1148/radiol.2018180547

13. Litjens G, Kooi T, Bejnordi BE et al (2017) A survey on deep learning in medical image analysis. Med Image Anal 42:60-88. https://doi.org/10.1016/j.media.2017.07.005

14. Chartrand G, Cheng PM, Vorontsov E et al (2017) Deep learning: a primer for radiologists. RadioGraphics 37:2113-2131. https:// doi.org/10.1148/rg.2017170077

15. Roth HR, Lu L, Seff A et al (2014) A new 2.5D representation for lymph node detection using random sets of deep convolutional neural network observations. Med Image Comput Comput Assist Interv 17:520-527

16. Längkvist M, Jendeberg J, Thunberg P et al (2018) Computer aided detection of ureteral stones in thin slice computed tomography volumes using Convolutional Neural Networks. Comput Biol Med. https://doi.org/10.1016/j.compbiomed.2018.04.021
17. Parakh A, Lee H, Lee JH et al (2019) Urinary stone detection on CT images using deep convolutional neural networks: evaluation of model performance and generalization. Radiol Artif Intell 1:e180066. https://doi.org/10.1148/ryai.2019180066

18. Guest AR, Cohan RH, Korobkin M et al (2001) Assessment of the clinical utility of the rim and comet-tail signs in differentiating ureteral stones from phleboliths. Am J Roentgenol 177:12851291. https://doi.org/10.2214/ajr.177.6.1771285

19. Traubici J, Neitlich JD, Smith RC (1999) Distinguishing pelvic phleboliths from distal ureteral stones on routine unenhanced helical CT: is there a radiolucent center? Am J Roentgenol 172:13-17. https://doi.org/10.2214/ajr.172.1.9888730

20. Nestler T, Haneder S, Hokamp NG (2019) Modern imaging techniques in urinary stone disease. Curr Opin Urol 29:81-88. https ://doi.org/10.1097/MOU.0000000000000572

Publisher's Note Springer Nature remains neutral with regard to jurisdictional claims in published maps and institutional affiliations. 